\author{
Military Technical College \\ Kobry El-Kobbah, \\ Cairo, Egypt
}

\section{$7^{\text {th }}$ International Conference on Electrical Engineering ICEENG 2010}

\title{
Stability and Control Augmentation Systems for an Aerosonde UAV
}

\author{
By \\ K.N.Mobariz* \\ M.S.Sayed* \\ A.M.Youssef* \\ M.A.El-Rahman**
}

\section{$\underline{\text { Abstract: }}$}

Unmanned aerial vehicles suffer from inherent instabilities in the pitch axis due to their small size and the lack of pilot feedback. Therefore a flight control system is needed whose primary function consists of artificial stabilization of the aircraft. This system is known as pitch axis stability augmentation system. Control augmentation system is a common part of modern airplane control and is best characterized as a form of tracking control. In this paper, a flight control system is to be designed that augments both stability and control for an unmanned aerial vehicle called Aerosonde. This control system is designed using Linear Quadratic Gaussian technique. The performance of the control system is compared with the performance of a classical PID controller in terms of input tracking and disturbance rejection properties.

\section{Keywords:}

Stability augmentation system (SAS), control augmentation system (CAS), linear quadratic Gaussian (LQG), unmanned aerial vehicle (UAV).

* Egyptian Armed Forces

** Ass. Prof. electrical power Engineering, Ain Shams University

*** College of Engineering, Cairo University, Cairo, Egypt 


\section{Introduction:}

Unmanned air vehicles (UAVs) have been a very active area of research since they are indispensable for various applications where human intervention is considered difficult or dangerous. It is well known that UAVs suffer from inherent instabilities in the pitch axis due to their small size and the lack of pilot feedback.

To enhance the oscillatory dynamic stability of the aircraft, an automatic control system is necessary to provide suitable damping and natural frequencies for better aircraft responsiveness and smoother disturbance rejection. Such control system is known as Stability Augmentation Systems (SAS). This system generally utilizes pitch rate in the control laws. The sensors commonly utilized to measure this parameter are the rate gyros.

Control augmentation system (CAS) is a common part of modern aircraft control and is best characterized as a form of tracking control. Therefore, to perform tasks such as precision tracking of targets, a specialized CAS is needed known as a pitch-rate CAS. The controlled variable of such system is the pitch-rate $(Q)$, which is required to follow a pilot's joystick command. It has been found that a deadbeat response to pitch-rate commands is well suited to the task. This system is conventionally designed for the longitudinal dynamics.

In the early 60 's, Kalman derived analytical solutions to linear quadratic optimal control problem for deterministic systems (Linear Quadratic Regulator) and for Gaussian stochastic systems (Linear Quadratic Gaussian). After the emergence of the reports of successful use of optimal control concepts in oil industries in the late 70's and early 80 's, a rapid development in the optimal control theories and a bringing forth of the work of Kalman have been seen. To date, this remains one of the most influential discoveries of the modern control era.

In this paper, a flight control system is to be designed that augments both stability and control for an UAV called Aerosonde. This control system is designed using Linear Quadratic Gaussian (LQG) technique. The performance of the control system is compared with the performance of a classical PID controller in terms of input tracking and disturbance rejection properties.

This paper is organized as follows: In Section 2, a mathematical description of the aircraft model movement is introduced. Section 3 describes the underlying aircraft stability and control augmentation systems (SCAS) and poses the performance requirements imposed on them. Section 4 gives a brief description of the classical PID tuning method and the LQG control theory used for the control system design. Designing procedures and simulations of the closed-loop system with both design techniques are presented in Section 5 and finally, this paper concludes with a brief summary in Section 6. 


\section{Mathematical Model of UAV:}

A generalized full six degrees of freedom nonlinear mathematical model of a rigid symmetric aircraft can be written as follows $[1,2]$ :

$$
\begin{aligned}
& X_{A}+X_{G}+X_{T}=m(\dot{U}+W Q-V R) \\
& Z_{A}+Z_{G}+Z_{T}=m(\dot{W}+V P-U Q) \\
& Y_{A}+Y_{G}+Y_{T}=m(\dot{V}+U R-W P) \\
& L_{A}+L_{G}+L_{T}=\dot{P} I_{x x}+Q R\left(I_{z z}-I_{y y}\right)-(\dot{R}+P Q) I_{x z} \\
& M_{A}+M_{G}+M_{T}=\dot{Q} I_{y y}+P R\left(I_{x x}-I_{z z}\right)+\left(P^{2}-R^{2}\right) I_{x z} \\
& N_{A}+N_{G}+N_{T}=\dot{R} I_{z z}+P Q\left(I_{v v}-I_{x x}\right)-(\dot{P}+Q R) I_{x z} \\
& \dot{\phi}=P+Q \sin \phi \tan \theta+R \cos \phi \tan \theta \\
& \dot{\theta}=Q \cos \phi-R \sin \phi \\
& \dot{\psi}=Q \sin \phi \sec \theta+R \cos \phi \sec \theta
\end{aligned}
$$

According to the aerodynamic stability and control derivatives associated to the Aerosonde UAV [3], the nonlinear aircraft model is linearized about the trim condition (straight and level flight: $\mathrm{V}_{\mathrm{T}}=25 \mathrm{~m} / \mathrm{s}, \mathrm{h}=300 \mathrm{~m}, \varphi=0$ ). The resulting linear model is then decoupled into longitudinal and lateral directional plants. The longitudinal plant model is given as follows:

$$
\begin{aligned}
& {\left[\begin{array}{c}
\dot{U} \\
\dot{W} \\
\dot{Q} \\
\dot{\theta}
\end{array}\right]=\left[\begin{array}{cccc}
-0.2368 & 0.5319 & -1.2158 & -9.8100 \\
-0.5665 & -4.4286 & 24.3798 & -0.4856 \\
0.4310 & -4.7929 & -5.1089 & 0 \\
0 & 0 & 1 & 0
\end{array}\right]\left[\begin{array}{c}
U \\
W \\
Q \\
\theta
\end{array}\right]+\left[\begin{array}{c}
0.3504 \\
-2.5231 \\
-34.8697 \\
0
\end{array}\right]\left[\delta_{\epsilon}\right]} \\
& C=Q=\left[\begin{array}{llll}
0 & 0 & 1 & 0
\end{array}\right]
\end{aligned}
$$

With the reference to Table (1), the eigenvalues of longitudinal state matrix reveal that there are two sets of complex poles; the low frequency lightly damped set, called phugoid mode, mainly affects pitch attitude $\theta$ and forward speed $U$, and the high frequency well damped set called short period mode, mostly affects the transient responses in normal speed $W$ and pitch rate $Q$. The uncontrolled open-loop step response of the pitch rate to the elevator input and its corresponding root-locus plot are 
plotted in Figures $(1,2)$. The step response confirms that the system is lightly damped, and the root locus plot shows that the system goes unstable quickly as the gain exceeds 0.274 .

Table (1): Characteristics of longitudinal dynamic stability

\begin{tabular}{|c|c|c|c|}
\hline Mode & Eigenvalues & $\begin{array}{c}\text { Damping Ratio } \\
\boldsymbol{\zeta}\end{array}$ & $\begin{array}{c}\text { Natural } \\
\text { Frequency } \\
\boldsymbol{\omega}_{\boldsymbol{n}}\end{array}$ \\
\hline Short-period & $-4.77+\mathrm{j} 10.8$ & 0.403 & 11.8 \\
\hline Phugoid & $-0.119+\mathrm{j} 0.554$ & 0.211 & 0.567 \\
\hline
\end{tabular}

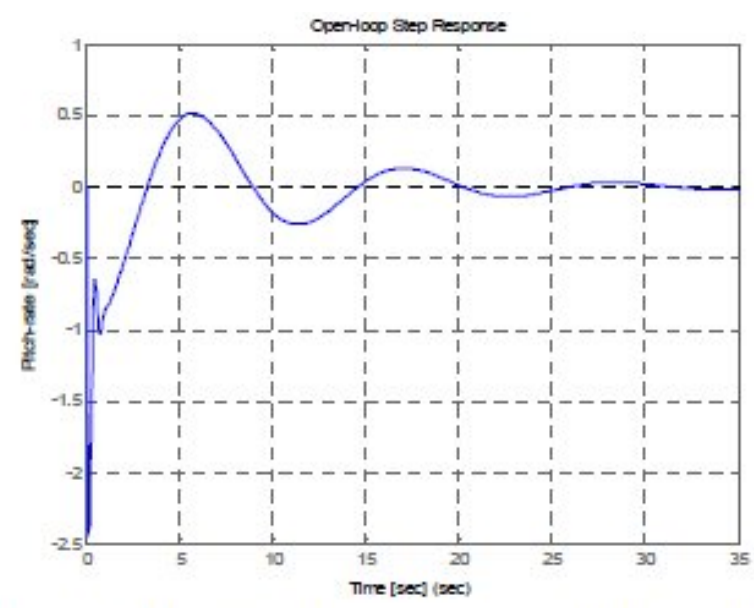

Figure (1): Open-loop response of the pitch rate to a step in the elevator

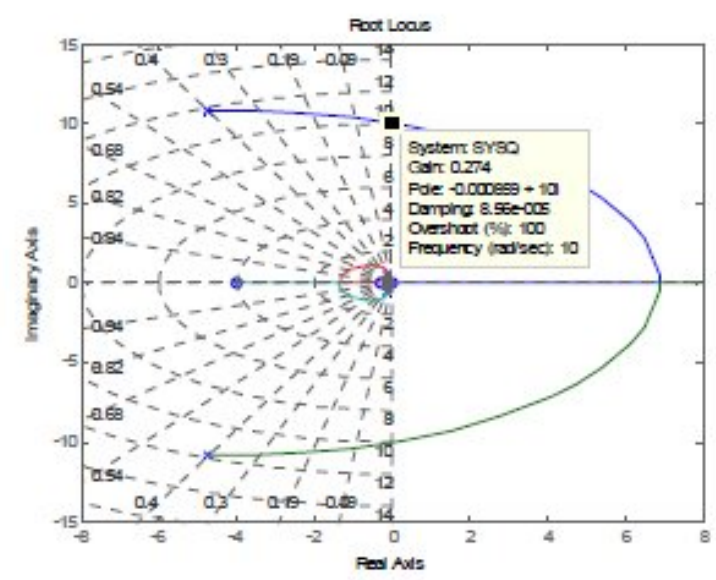

Figure (2): Root locus for the open-loop

\section{Longitudinal SCAS:}

It is well known that for achieving requirements on both set-point tracking and disturbance rejection simultaneously, it is convenient to use a two degrees-of-freedom (TDOF) controller. The control system is divided into two control loops as in Figure (3). The pitch-rate SAS inner loop, which is designed to increase the damping of the shortperiod oscillation. This can be accomplished by adding an inner feedback loop utilizing a rate gyro for sensing the pitch-rate controlled variable. The CAS outer loop consists of the feedback of the output $Q$. The error difference between $Q_{\text {com }}$ and $Q$ is fed to a PID controller to improve tracking performance. Since there is a sign change present inherently in the aircraft dynamics associated with the relationship of the pitch-rate to elevator deflection, the feedback signal is added to the SAS command voltage signal. 


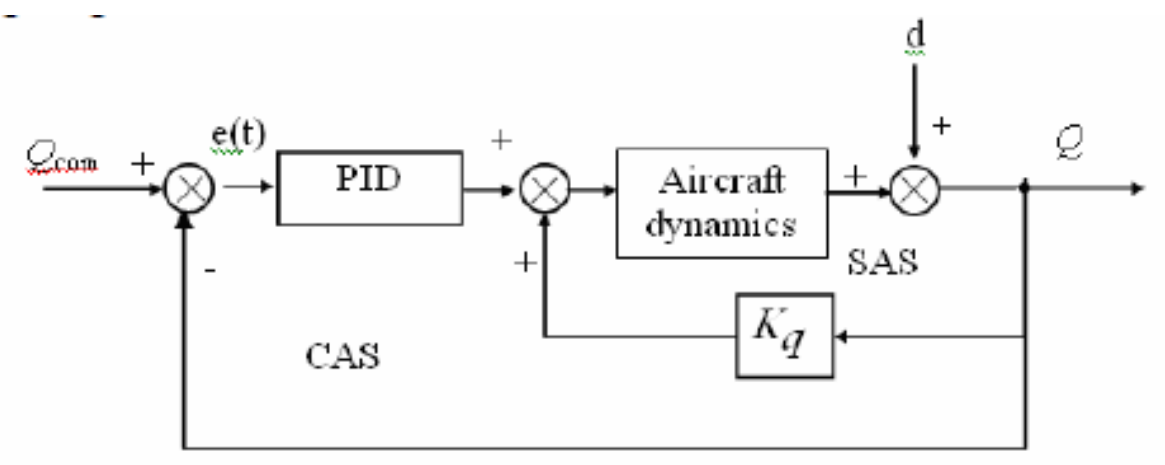

Figure (3): Pitch-rate stability and control augmentation systems

The overall performance objective is to track pitch-rate commands with predicted Level 1 handling qualities and desired time-domain response behavior. Table (2) summarizes the boundaries of the handling qualities that must be Level 1. In this application, the more stringent 'Good Level 1' boundaries are used.

Table (2): Handling quality boundaries

\begin{tabular}{|c|c|c|}
\hline HQs & Level 1 & Good Level 1 \\
\hline Short period & $0.35<\zeta_{s p}<1.35$ & $0.7<\zeta_{s p}<1.35$ \\
\hline Settling time & $2 \%$ within $3 \mathrm{sec}$ & $1 \%$ within $3 \mathrm{sec}$ \\
\hline
\end{tabular}

For the command tracking specification, the designed control system must satisfy a step response with rise time $(90 \%$ of the final value) $<0.5 \mathrm{sec}$ and overshoot $<5 \%$ is required.

Disturbance rejection is an important objective in addition to command tracking. Therefore, the designed controllers must satisfy the requirements imposed on disturbance rejection for disturbances enter directly at the plant output, which represent the wind gusts. The assumption of white noise disturbances was not always relevant or meaningful to practicing control engineers. As in case of the vertical wind gust $\mathrm{w}_{\mathrm{g}}$, it is found that $\mathrm{w}_{\mathrm{g}}$ is not a white noise, but it has a spectral density given in Dryden form as[4]:

$$
\phi_{\text {wind }}(\omega)=2 L \sigma^{2} \frac{1+3 L^{2} \omega^{2}}{\left(1+L^{2} \omega^{2}\right)^{2}}
$$


where $\omega$ is the frequency in $[\mathrm{rad} / \mathrm{sec}], \sigma$ is the turbulence intensity and $L$ is the turbulence scale length divided by true airspeed. Hence, the authors of the references [4, 5] tried to determine shaping filters driven by a white noise and generate a noise with spectral density given by Equation (3). The generated disturbance noise is found to be always less than unity in magnitude. In this work, the worst-case behavior of a system is analyzed by considering disturbance of magnitude equals to unity, and hence the disturbance rejection specification is that the output response to a unit step disturbance should remain within the range $[-1,1]$ at all times, and return to zero as quickly as possible $(90 \%$ is rejected within $3 \mathrm{sec})$.

\section{Controller Design Techniques:}

This section covers a review of both PID and LQG controllers design methods.

\subsection{PID tuning by Ziegler and Nichols:}

The Ziegler-Nichols (Z-N) design methods are the most popular heuristic methods used in process control for determining the parameters of a PID controller. Although these methods date back to early 40s for analogue controllers, they are still appropriate for modern digital control systems. Ziegler and Nichols have proposed two methods for tuning the PID called: the continuous cycling method and the process reaction curve method [6]. A brief review of the first method is given, which is used in next section. It is assumed that the controller is parameterized as follows:

$$
\begin{aligned}
G_{c}(s) & =K_{p}+\frac{K_{i}}{s}+K_{d} s \\
& =\mathrm{K}_{\mathrm{c}}\left(1+\frac{\mathrm{T}_{\mathrm{i}}}{\mathrm{s}}+\mathrm{T}_{\mathrm{d}} \mathrm{s}\right)
\end{aligned}
$$

where $\mathrm{K}_{\mathrm{c}} \approx K_{p}$ is the proportional gain, $\mathrm{T}_{\mathrm{i}}=\frac{K_{p}}{K_{i}}$ and $\mathrm{T}_{\mathrm{d}}=\frac{K_{d}}{K_{p}}$ are the integral and derivative

time constant, respectively. In the continuous cycling method, the controller gain is increased until a sustained oscillation takes place at gain $\mathrm{Ku}$. If the corresponding period of oscillation be $\mathrm{Tu}$, then the parameters of the PID controller, as suggested by Ziegler and Nichols, can be calculated using Table (3). 
Table (3): Z-N (continuous cycling method) tuning rules

\begin{tabular}{|c|c|c|c|}
\hline \multirow{2}{*}{ Controller } & \multicolumn{3}{|c|}{ Parameters } \\
\hline & $\mathbf{K}_{\mathrm{c}}$ & $\mathbf{T}_{\mathrm{i}}$ & $\mathbf{T}_{\mathbf{d}}$ \\
\hline P & $0.5 \mathrm{~K}_{\mathrm{u}}$ & & \\
\hline PI & $0.45 \mathrm{~K}_{\mathrm{u}}$ & $\mathrm{T}_{\mathrm{u}} / 1.2$ & \\
\hline PID & $0.6 \mathrm{~K}_{\mathrm{u}}$ & $0.5 \mathrm{~T}_{\mathrm{u}}$ & $\mathrm{T}_{\mathrm{u}} / 8$ \\
\hline
\end{tabular}

\subsection{LQG Controller:}

In this subsection, a brief review of the LQG controller design is given. The LQG controller is the combination of the Linear Quadratic Regulator (LQR) and Kalman filter estimator. By means of the separation principle, we are able to design the LQR controller followed by a state estimator.

\subsubsection{LQR:}

The LQR problem, where all the states are measurable, is a deterministic initial value problem. For a controllable and observable system:

$$
\dot{x}=A x+B u
$$

with $x \in \mathrm{R}^{\mathrm{n}}, u \in \mathrm{R}^{\mathrm{m}}$ and with a non-zero initial state $x(0)$. The controls will be state feedback of the form

$$
u(t)=-K x(t)
$$

where $K$ is called Kalman gain. The objective is to find the gain $K$ so that the control input $u(t)$ can take the system to the zero state $(x=0)$ in an optimal manner, i.e. by minimizing the deterministic performance index

$$
J=\frac{1}{2} \int_{0}^{\infty}\left(x^{T} Q_{w} x+u^{T} R_{w} u\right) d t
$$

where $Q_{w}$ and $R_{w}$ are appropriately chosen weighting matrices (design parameters) such that $Q_{w}=Q_{w}{ }^{T} \geq 0$ and $R_{w}=R_{w}{ }^{T}>0$.

The optimal solution is given by:

$$
K=R_{w}^{-1} B^{T} P
$$

where $P=P^{T} \geq 0$ is the unique positive semi-definite solution of the algebraic Riccati equation: 


$$
A^{T} P+P A-P B R_{w}^{-1} B^{T} P+Q_{w}=0
$$

If the system $(A, B)$ is controllable and $\left(\sqrt{Q_{w}}, A\right)$ is observable, the Kalman gain $K$ is guaranteed to stabilize the plant and yield a global minimum value for performance index.

\subsubsection{Kalman filter:}

In practice, the assumption that all state variables are measurable is unlikely to be true, therefore a Kalman filter has to be designed for state estimation. Given the following state and output equations

$$
\begin{aligned}
& \dot{x}=A x+B u+F w \\
& y=C x+v
\end{aligned}
$$

where $w$ and $v$ are process and sensor noise respectively, we introduce a state estimator (11)

$$
\hat{\dot{x}}=A \hat{x}+B u+L(y-C \hat{x})
$$

where $\hat{x}$ is an estimate of $x$. The overall state equations may be obtained from (10) and (11) as

$$
\left[\begin{array}{l}
\dot{x} \\
\hat{x}
\end{array}\right]=\left[\begin{array}{cc}
A & 0 \\
L C & A-L C
\end{array}\right]\left[\begin{array}{l}
x \\
\hat{x}
\end{array}\right]+\left[\begin{array}{lll}
B & F & 0 \\
B & 0 & L
\end{array}\right]\left[\begin{array}{l}
u \\
w \\
v
\end{array}\right]
$$

Let $S_{w}$ and $S_{v}$ be the spectral densities of zero mean white noise $w$ and $v$ respectively, the optimal estimation gain $L$ vector is given by

$$
L=P C^{T} S_{v}^{-1}
$$

in which, $P$ is the solution of the following equation:

$$
P A^{T}+A P+F S_{w} F^{T}-P C^{T} S_{v}^{-1} C P=0
$$

\subsubsection{LQG:}

By combining the Kalman filter and LQR control, the estimated state variables can be used to replace the unmeasurable state $x$ in (6) to give

$$
u(t)=-K \hat{x}(t)+r
$$

where $r$ is the reference input signal and $\hat{x}$ is the estimated state obtained using the Kalman filter. The design objective is to minimize a quadratic cost function given by the limit

$$
J_{L Q G}=\lim _{T \rightarrow \infty} \int_{0}^{T}\left\{\left[\begin{array}{ll}
x^{T} & u^{T}
\end{array}\right] Z\left[\begin{array}{l}
x \\
u
\end{array}\right]\right\} d t
$$


By combining the Kalman filter and LQR equations, we obtain the following augmented matrix:

$$
\left[\begin{array}{c}
\dot{x} \\
\hat{\dot{x}}
\end{array}\right]=\left[\begin{array}{cc}
A & -B K \\
L C & (A-L C-B K)
\end{array}\right]\left[\begin{array}{l}
x \\
\hat{x}
\end{array}\right]+\left[\begin{array}{ccc}
B & F & 0 \\
B & 0 & L
\end{array}\right]\left[\begin{array}{l}
r \\
w \\
v
\end{array}\right]
$$

\section{SCAS Designing and Simulation Results:}

This section shows the design of the pitch-rate SCAS for the Aerosonde UAV by means of PID and LQG. The design approach is based upon the natural separation of the two control loops; the inner loop SAS and the outer loop CAS.

\subsection{SCAS Designing bv PID:}

SAS design is carried out by choosing $K_{q}$. This first loop is closed by placing the poles with a damping ratio of 0.7 . Root locus analysis shows that the dominant actuator pole interacts with the natural poles. Figure (4) shows that the poles follow an approximately circular arc as the pitch-rate feedback is increased until $K_{q}$ reaches 0.58 . With larger values, a new higher-frequency oscillatory mode is created, so large values of $K_{q}$ are not appropriate. Selecting a low value for $K_{q},\left(K_{q}=0.3\right)$, places the short-period eigenvalues at $s=-10 \pm \mathrm{j} 9.09$. This corresponds to a natural frequency of $13.5 \mathrm{rad} / \mathrm{sec}$ and a damping ratio of 0.74 .

Once the SAS design is done, the Z-N continuous cycling method is used for determining the PID parameters for the CAS design. From the analysis of the root locus for the open-loop transfer function; Figure (2), the sustained oscillation takes place at gain $\mathrm{Ku}=0.274$, and its corresponding frequency and period of oscillation are $\omega_{\mathrm{u}}=10$ $\mathrm{rad} / \mathrm{sec}$ and $\mathrm{T}_{\mathrm{u}}=2 \pi / \omega_{\mathrm{u}}=2 \pi / 10=0.628 \mathrm{sec}$, respectively. Placing the values of $\mathrm{K}_{\mathrm{u}}$ and $\mathrm{Tu}$ into Z-N Table (3), the following PID controller parameters are calculated: $K_{p}=$ $0.1644, K_{i}=0.5235, K_{d}=0.0129$. 


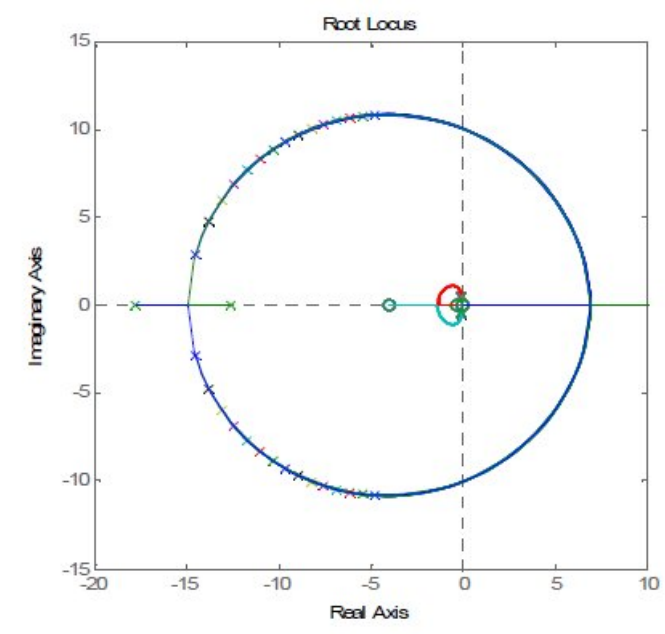

Figure (4): Effect of varying $K_{q}$ by root-locus plot

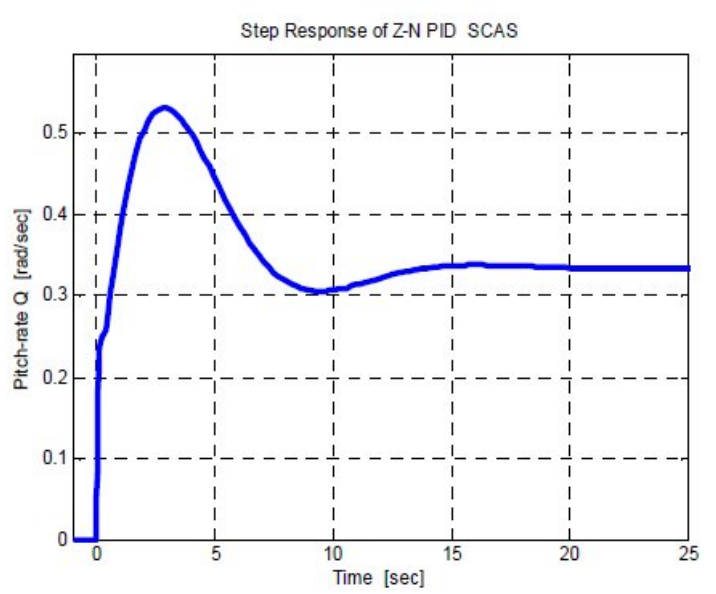

Figure (5):Step response with the Z-N PID

The step response of the Z-N PID SCAS is shown in Figure (5). It is obvious that the response behaves badly where the steady-state value reaches 0.334 . Therefore, the PID parameters are retuned using the genetic algorithm (GA) optimization technique under constraints of performance specifications. The GA PID parameters obtained are $K_{p}=$ $1.486, K_{i}=62.06, K_{d}=0.4023$ and the corresponding step is shown in Figure (6). The designed control system satisfies the requirements; where the rise time is $0.011 \mathrm{sec}$ and with no overshoot. Figure (7) shows the output response to a unit step change in the disturbance, applied directly at the plant output, where $50 \%$ of the disturbance is rejected within $0.0038 \mathrm{sec}$ and $90 \%$ is rejected within $0.015 \mathrm{sec}$.

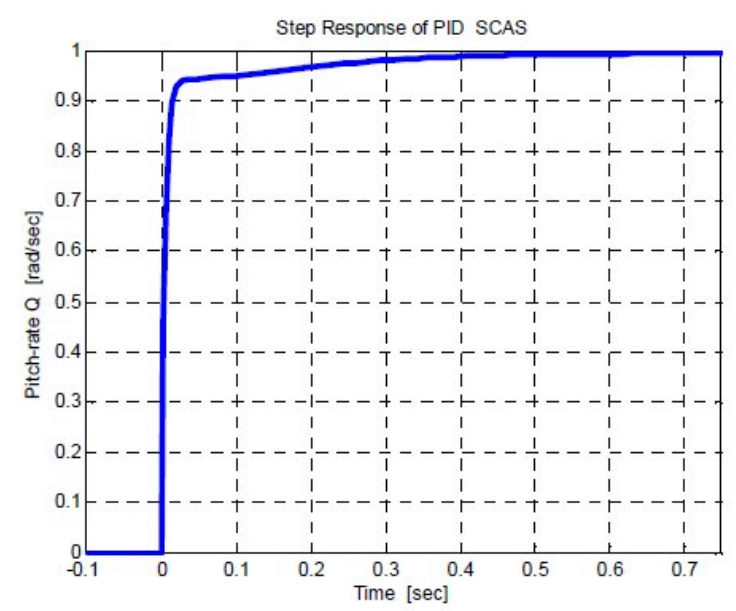

Figure (6): Step response with the GA PID controller

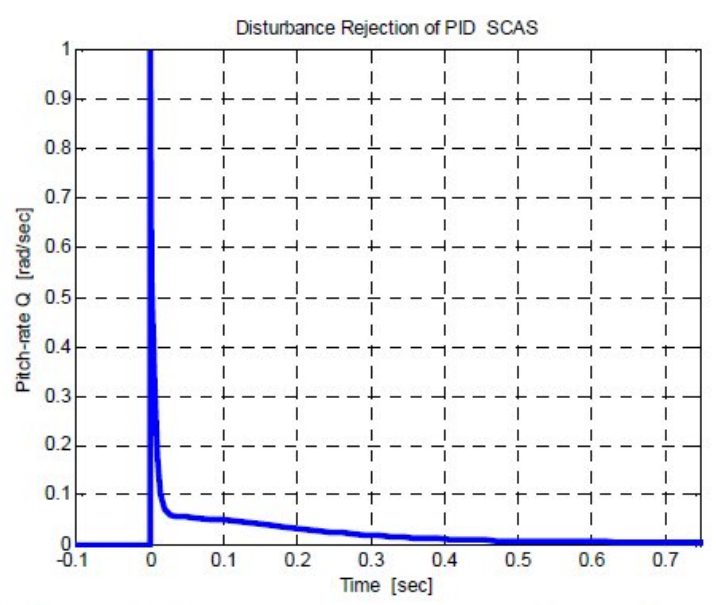

Figure (7):Output response to disturbance input 


\subsection{SCAS Designing by LQG:}

In this subsection, we consider time domain optimal control approaches using LQG controller.

\subsubsection{LQR Controller:}

Starting from the design of the LQR controller, the root-locus is used to choose the best relative weighting between $Q_{w}$ and $R_{w}$ matrices. The root-locus plot of the plant using different values for $Q_{w}$ and for $R_{w}=1.25$ is shown in Figure(8).

It is clear that as $\mathrm{Qw}$ increases from zero, the damping ratio and the natural frequency increases until $\mathrm{Qw}$ reaches 0.5 . Above this value, the two imaginary poles of the shortperiod immigrate to the real axis. Our selected values for $\mathrm{Qw}$ and $\mathrm{Rw}$ are chosen to be 0.2 and 1.25 , respectively, which place the short-period eigenvalues at $\mathrm{s}=-8.77 \pm \mathrm{j}$ 8.61. This corresponds to a natural frequency of $12.3 \mathrm{rad} / \mathrm{sec}$ and a damping ratio of 0.713 . The selected values of $\mathrm{Qw}$ and $\mathrm{Rw}$ results in a controllable system (A, B) and an observable

$\left(\sqrt{Q_{w}}, A\right)$, and Kalman gain $K$ is guaranteed to stabilize the plant which is given as:

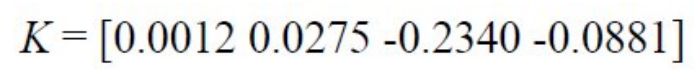

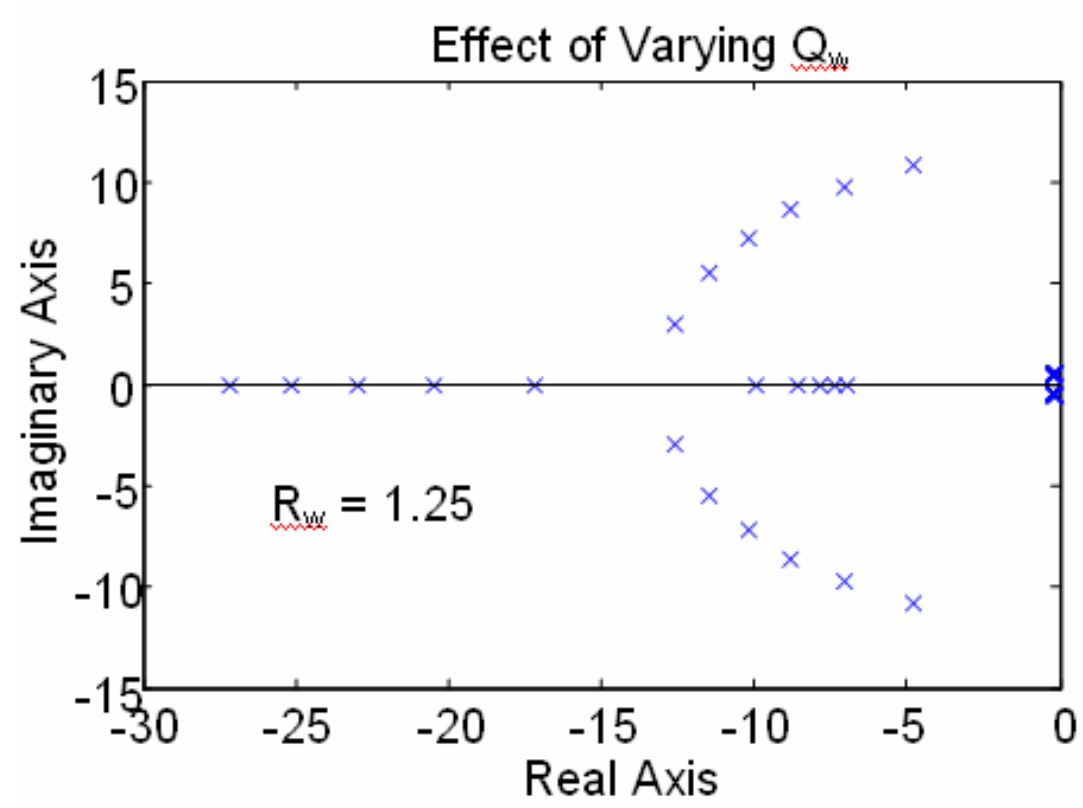

Figure (8): Choosing the best relative weighting between $Q w$ and $R w$ 


\subsubsection{Kalman Filter:}

The state feedback gain matrix requires all the states to be measurable. However, only one output, pitch-rate Q, can be measured. So the second step for designing the LQG controller is to design the Kalman filter. After some iterations, the selected values for $\mathrm{Sw}$ and $\mathrm{Sv}$ are given by:

$$
S_{w}=\left[\begin{array}{cccc}
0 & 0 & 0 & 0 \\
0 & 0 & 0 & 0 \\
0 & 0 & 0.0008 & 0 \\
0 & 0 & 0 & 0
\end{array}\right] \quad S_{v}=[0.0001]
$$

which yield the Kalman gain matrix $L$ and the solution to the algebraic Riccati equation $P$ as follows:

$$
L=\left[\begin{array}{c}
6.9650 \\
11.890 \\
24.738 \\
-8.8 \mathrm{e}-016
\end{array}\right] \quad P=\left[\begin{array}{cccr}
3.0643 \mathrm{e}-002 & -2.3157 \mathrm{e}-003 & 6.9650 \mathrm{e}-004 & -1.1983 \mathrm{e}-003 \\
-2.3157 \mathrm{e}-003 & 5.2584 \mathrm{e}-003 & 1.1890 \mathrm{e}-003 & 4.0838 \mathrm{e}-004 \\
6.9650 \mathrm{e}-004 & 1.1890 \mathrm{e}-003 & 2.4738 \mathrm{e}-003 & -8.8091 \mathrm{e}-020 \\
-1.1983 \mathrm{e}-003 & 4.0838 \mathrm{e}-004 & -8.8091 \mathrm{e}-020 & 1.2207 \mathrm{e}-004
\end{array}\right]
$$

Since the system $(C, A)$ is observable and $\left(A, \sqrt{S_{w}}\right)$ is controllable, then the error system ( $\left.A_{0}=A-L C\right)$ is asymptotically stable [4]. This means that the estimation error vanishes with time and then $\hat{x}(t)$ will approach $x(t)$.

\subsubsection{Prefilter Design:}

After designing the optimal dynamic regulator using LQG control technique, the remaining stage is to design a prefilter to improve the tracking performance. Figure (9) shows the block diagram of the pitch-rate CAS where a PID controller, as a prefilter, is cascaded to the SAS. Then a unity-gain outer loop is made to feed the performance output, pitch-rate $Q$, back and subtract it from the reference command, thus the input to the prefilter is the tracking error $e(t)$.

The GA optimization technique under constraints of performance specifications is used for obtaining the optimal PID parameters. The GA PID parameters obtained are $K_{p}=$ 15.47, $K_{i}=117.3, K_{d}=0.4019$ and the corresponding step is shown in Figure (10). The designed control system satisfies the requirements; where the rise time is $0.003 \mathrm{sec}$ and with no overshoot. Figure (11) shows the output response to a unit step change in the 
disturbance, applied directly at the plant output, where $50 \%$ of the disturbance is rejected within $0.0009 \mathrm{sec}$ and $90 \%$ is rejected within $0.0029 \mathrm{sec}$.

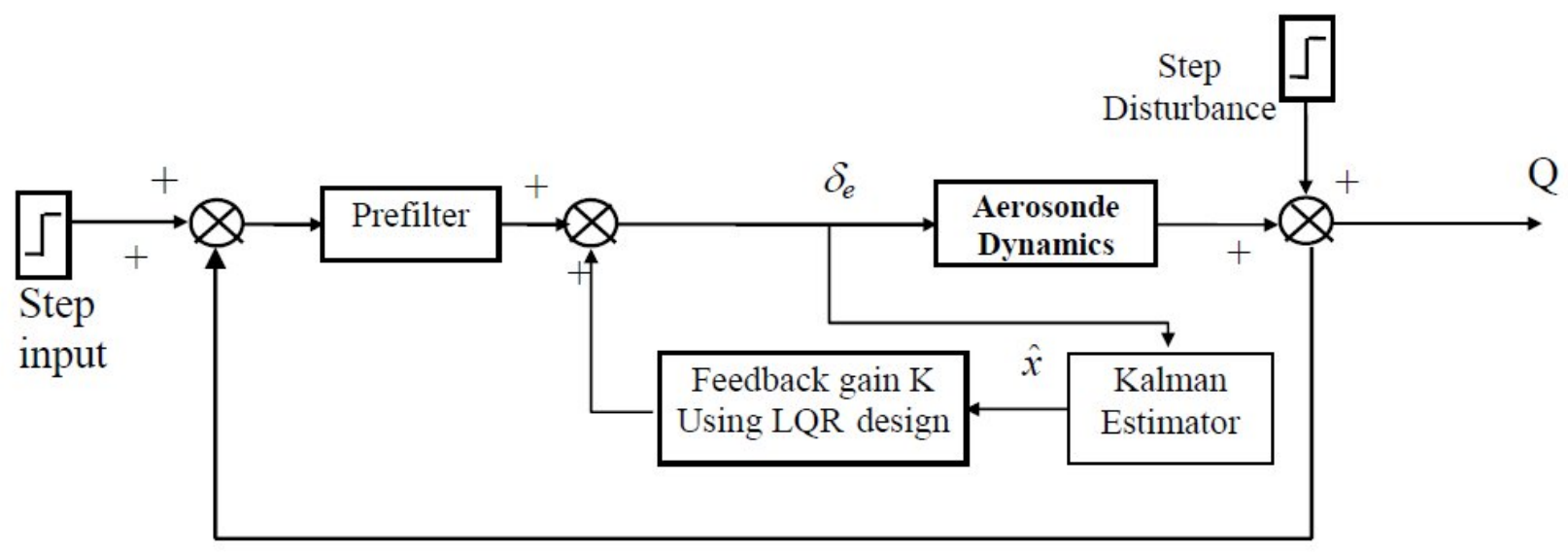

Figure (9): Pitch-rate control augmentation system using $L Q G$ controller

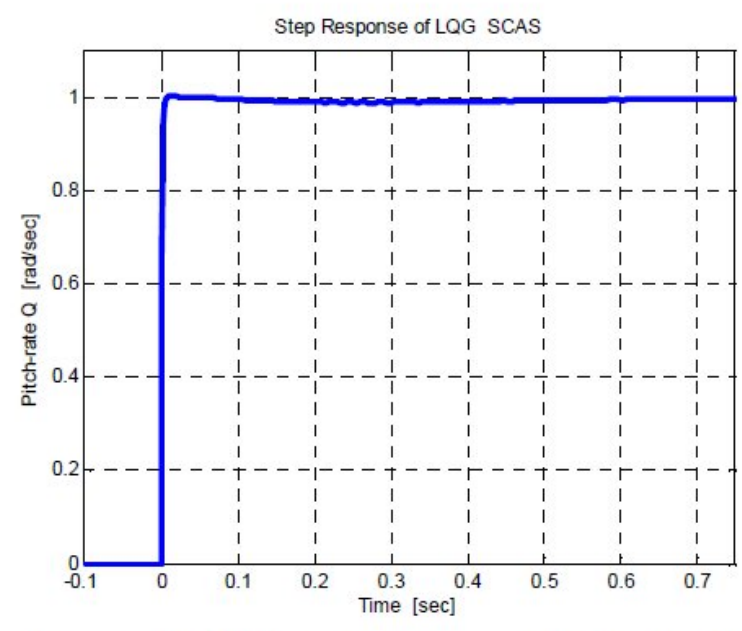

Figure (10):Step response with the LQG controller

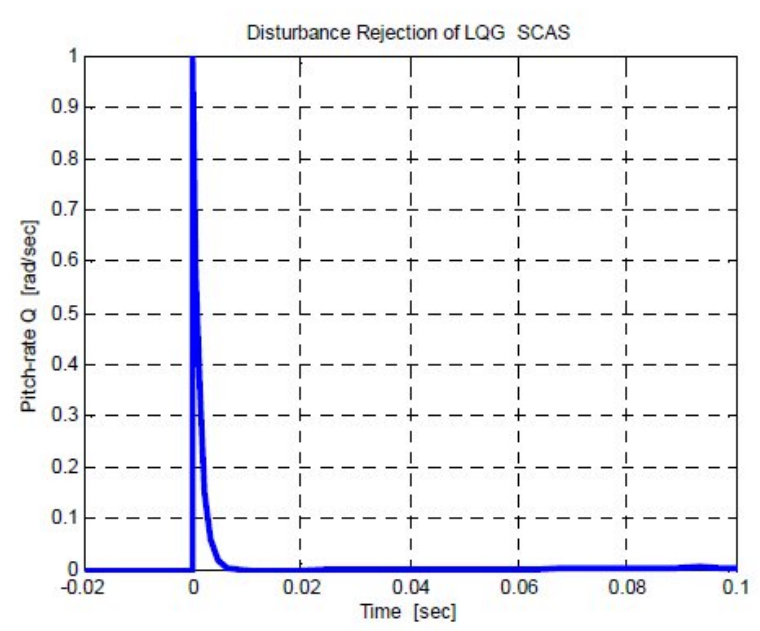

Figure (11):Output response to disturbance input

\section{Conclusions:}

In this paper, a flight control system is designed that augments both stability and control for Aerosonde UAV. This control system is first designed using classical Z-N tuning algorithm for PID controller, which showed bad behavior. The PID parameters are then optimized using GA optimization technique under performance specifications. Next, the control system is designed using LQG technique and is compared with the performance 
of the PID controller. Although for the two techniques, the design objectives were satisfied including good tracking and disturbance rejection, the LQG is proved to have better performances.

\section{References:}

[1] Etkin, B., Dynamics of Flight: Stability and Control, John Wiley \& sons, New York, 1982.

[2] McRuer, D., I. Ashkenas and D. Graham, Aircraft Dynamics and Automatic Control, Princeton, 1973.

[3] Flight Dynamic web page: www.u-dynamics.com

[4] Stevens, B.L. and F.L. Lewis, Aircraft Control and Simulation, John Wiley \& sons, 1992.

[5] McLean, D., Automatic Flight Control Systems, Prentice-Hall, UK, 1990.

[6] Mukhtiar, A. U., D.J. Murray-Smith, and Syed F. A., Design and Tuning of fixed structure PID Controllers A Survey, Technical Report CSC-96016, Faculty of Engineering, Glasgow University, Scotland, 1996.

\section{Nomenclatures:}

$\begin{array}{ll}V_{T} & \text { True airspeed } \\ h & \text { Altitude } \\ \delta_{e} & \text { Elevator deflection } \\ I x x, I y y, I z z & \text { Moment of inertia about each axis } \\ I y z, I z x, I x y & \text { product of inertia } \\ \theta \phi \psi & \text { Euler angles (pitch, roll, yaw) } \\ X_{A}, Y_{A}, Z_{A} & \text { aerodynamic force components (axial, side, normal) } \\ X_{G}, Y_{G}, Z_{G} & \text { gravity force components (axial, side, normal) } \\ X_{T}, Y_{T}, Z_{T} & \text { propulsive force components (axial, side, normal) } \\ L_{A}, M_{A}, N_{A} & \text { aerodynamic moment components (rolling, pitching, yawing) } \\ L_{G}, M_{G}, N_{G} & \text { gravity moment components (rolling, pitching, yawing) } \\ L_{T}, M_{T}, N_{T} & \text { propulsive moment components (rolling, pitching, yawing) } \\ U, V, W & \text { total linear velocity components (axial, side, normal) } \\ M & \text { mass } \\ P, Q, R & \text { total angular velocity components (roll rate, pitch rate, yaw rate) }\end{array}$

